\title{
Editorial: Asteroseismology in the Kepler Era
}

\author{
Anthony E. Lynas-Gray ${ }^{1,2,3}$, Andrzej S. Baran ${ }^{4,5,6 *}$ and Karen Kinemuchi ${ }^{7}$ \\ ${ }^{1}$ University College London, London, United Kingdom, ${ }^{2}$ University of Oxford, Oxford, United Kingdom, ${ }^{3}$ University of the \\ Western Cape, Bellville, South Africa, ${ }^{4}$ ARDASTELLA Research Group, Institute of Physics, Pedagogical University of Kraków, \\ Kraków, Poland, ${ }^{5}$ Department of Physical Science, Embry-Riddle Aeronautical University, Daytona Beach, FL, United States, \\ ${ }^{6}$ Department of Physics, Astronomy, and Materials Science, Missouri State University, Springfield, MO, United States, ${ }^{7}$ New \\ Mexico State University, Las Cruces, NM, United States
}

Keywords: kepler spacecraft, pulsating stars, asteroseismology, space photometry, fourier technique

\section{Editorial on the Research Topic}

\section{Asteroseismology in the Kepler Era}

The Kepler spacecraft, launched by the National Aeronautics and Space Administration (NASA) on 2009 March 7th, proved to be an important pioneering astronomical survey instrumental, making crucial discoveries during its primary mission over the ensuing 4 years. Looking longitudinally along the Local Spiral Arm in the general direction of Cygnus maximised the number of nearby (and therefore bright) stars that could be monitored almost continuously over the lifetime of the mission. A $0.95-\mathrm{m}$ mirror with detectors sensitive to wavelengths in range $430-890 \mathrm{~nm}$ offered the best prospect of detecting earth-sized planets with orbital periods of about one-year, transiting across the disks of solar-type stars. While the search for planets orbiting nearby stars, and those in habitable zones in particular, persuaded NASA to support the Kepler mission, it would be a mistake to suppose that asteroseismic studies constituted a secondary scientific objective. Stellar pulsation generates light-curve features which could be of the same order as the flux lost from a solar-type star when an earth-sized planet in its habitable zone transits across its disk; it is critical that the two are not confused. Asteroseismology therefore plays a role in the search for planets beyond the Solar System. Furthermore, the existence of a planetary system is likely to affect evolution and internal structure of the host star, thereby determining its asteroseismic properties. Searches for planets orbiting nearby stars, and asteroseismology of these objects, are therefore inextricably linked.

The Editors are extremely grateful to the authors and reviewers who have contributed papers on asteroseismology with the Kepler spacecraft. While only a tiny fraction of the scientific returns are presented, the sample is understood to be representative and serve to inspire and support future space missions which will achieve even more than the impressive results secured by the Kepler mission.

In particular, we look forward to theoretical developments which allow the full potential of asteroseismic observations to be realised.

\section{AUTHOR CONTRIBUTIONS}

This article was submitted to Stellar and Solar Physics,

a section of the journal Frontiers in Astronomy and Space

Received: 11 May 2021 Accepted: 11 August 2021 Published: 23 August 2021

Citation:

Lynas-Gray AE, Baran AS and Kinemuchi K (2021) Editorial: Asteroseismology in the Kepler Era. Front. Astron. Space Sci. 8:708266.

All authors listed have made a substantial, direct, and intellectual contribution to the work and approved it for publication.

Conflict of Interest: The authors declare that the research was conducted in the absence of any commercial or financial relationships that could be construed as a potential conflict of interest.

Publisher's Note: All claims expressed in this article are solely those of the authors and do not necessarily represent those of their affiliated organizations, or those of the publisher, the editors and the reviewers. Any product that may be evaluated in this article, or claim that may be made by its manufacturer, is not guaranteed or endorsed by the publisher.

Copyright $\odot 2021$ Lynas-Gray, Baran and Kinemuchi. This is an open-access article distributed under the terms of the Creative Commons Attribution License (CC BY). The use, distribution or reproduction in other forums is permitted, provided the original author(s) and the copyright owner(s) are credited and that the original publication in this journal is cited, in accordance with accepted academic practice. No use, distribution or reproduction is permitted which does not comply with these terms. 